\title{
ON TWO RESULTS OF J. DUGUNDJI ABOUT EXTENSIONS OF MAPS AND RETRACTIONS
}

\author{
H. STEINLEIN
}

\begin{abstract}
We give a short proof of Dugundji's result that spheres in infinite-dimensional normed spaces are absolute retracts.
\end{abstract}

In [1], Dugundji proved a very important extension theorem and used it to show the following fact:

TheOREM 1. Let $L$ be a normed linear space, $B:=\{x \in L \mid\|x\| \leqslant 1\}$ and $C:=\{x \in L \mid\|x\|=1\}$. If $C$ is not compact, then $C$ is a retract of $B$ (and hence an absolute retract).

An important consequence of Theorem 1 for the applications is that $C$ is contractible and an absolute extensor for metrizable spaces (cf. [3]). Another well-known consequence is the fact that closed balls in infinite-dimensional normed spaces do not have the fixed point property for continuous maps.

The aim of this paper is to give a slightly sharper version of the Dugundji extension theorem, which allows a very short and intuitive proof of Theorem 1.

Theorem 2. Let $X$ be a metric space, $A \subset X$ a closed subset, $D$ a dense subset of $A$, and $L$ a normed space (or more generally a locally convex space or an affine space of type $m$, cf. [2]). Then each continuous $f: A \rightarrow L$ has a continuous extension $F: X \rightarrow L$ with $F(X) \subset f(A) \cup$ [convex hull of $f(D)$ ].

The proof of Theorem 2 is the same as the one of the original theorem, if one chooses the points $a_{U} \in D$ (cf. [2, p. 188]).

Proof of Theorem 1 . Since $C$ is not compact, $L$ is infinite-dimensional. Hence $L$ has a proper dense linear subspace $L^{\prime}{ }^{1}$ By Theorem 2, applied to $X:=B, A:=C, D:=C \cap L^{\prime}$ and $f:=\mathrm{id}_{C}$, there exists a continuous map $F: B \rightarrow L$ with $\left.F\right|_{C}=\mathrm{id}_{C}$ and $F(B) \subset C \cup$ [convex hull of $C \cap L^{\prime}$ ] $=C \cup$

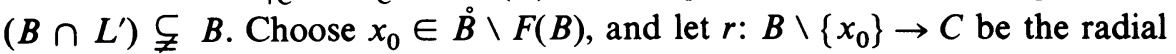
retraction. Then $r \circ F$ is a retraction from $B$ to $C$.

\footnotetext{
Received by the editors July 25, 1978 and, in revised form, December 30, 1978.

AMS (MOS) subject classifications (1970). Primary 54C15, 54C20; Secondary 47H10.

Key words and phrases. Tietze-Dugundji extension theorem, retract, fixed point property.

'One should recall that it is easy to prove the existence of such a space $L^{\prime}$ : Any unbounded real-valued function on a normalized Hamel basis of $L$ uniquely determines an unbounded linear functional $f$ on $L$. Then $L^{\prime}=$ [kernel of $\left.f\right]$ is not closed and codim $L^{\prime}=1$, and hence $L^{\prime}$ is dense.
} 
ACKNOWLeDGement. The author would like to thank Volker Eberhardt for a valuable discussion.

\section{REFERENCES}

1. J. Dugundji, An extension of Tietze's theorem, Pacific J. Math. 1 (1951), 353-367.

2. T. Topology, Allyn and Bacon, Boston, Mass., 1966.

3. S. T. Hu, Theory of retracts, Wayne State Univ. Press, Detroit, 1965.

Mathematisches Institut, Universttät MÜNChen, Theresienstr. 39, D 8000 MÜNChen 2, Federal Republic of Germany 